EUROPEAN ORGANIZATION FOR NUCLEAR RESEARCH

European Laboratory for Particle Physics

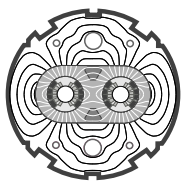

Large Hadron Collider Project

LHC Project Report 121

\title{
STABILITY DIAGRAMS FOR LANDAU DAMPING
}

Berg, J.S.; B Ruggiero, F.

\begin{abstract}
Coherent modes which are present when there is no incoherent tune spread may be absent when such a spread exists. Such modes are "Landau damped." There is instead an incoherent spectrum, a continuum of an infinite number of frequencies, which will decohere (filament), thus not leading to collective instabilities. A stability diagram indicates when Landau damping will be effective. It divides the effective impedance plane, or equivalently the plane of coherent frequency in the absence of tune spread, into regions. The region which contains $+i /$ infinity corresponds to instability. Thus, one can substitute a simpler computation (finding discrete eigenvalues) for a more complex computation (solving an eigenvalue system with both a discrete and a continuous eigenvalue spectrum). We present stability diagrams assuming a linear tune shift with amplitude, allowing tune spread in two transverse planes or in the longitudinal plane alone. When there is longitudinal tune spread, this can not be done exactly, and we describe approximations which make the computation tractable.
\end{abstract}

SL AP

Presented at 1997 Particle Accelerator Conference, Vancouver, 12-16 May 1997.

Administrative Secretariat

LHC Division

CERN

$\mathrm{CH}-1211$ Geneva 23

Switzerland

Geneva, 11 juin 1997 


\title{
Stability Diagrams for Landau Damping
}

\author{
J. Scott Berg and Francesco Ruggiero \\ European Laboratory for Particle Physics (CERN)
}

\section{Abstract}

Coherent modes which are present when there is no incoherent tune spread may be absent when such a spread exists. Such modes are "Landau damped." There is instead an incoherent spectrum, a continuum of an infinite number of frequencies, which will decohere (filament), thus not leading to collective instabilities. A stability diagram indicates when Landau damping will be effective. It divides the effective impedance plane, or equivalently the plane of coherent frequency in the absence of tune spread, into regions. The region which contains $+i \infty$ corresponds to instability. Thus, one can substitute a simpler computation (finding discrete eigenvalues) for a more complex computation (solving an eigenvalue system with both a discrete and a continuous eigenvalue spectrum). We present stability diagrams assuming a linear tune shift with amplitude, allowing tune spread in two transverse planes or in the longitudinal plane alone. When there is longitudinal tune spread, this can not be done exactly, and we describe approximations which make the computation tractable.

\section{INTRODUCTION}

This paper discusses stability diagrams for Landau damping in two situations: Landau damping of transverse oscillations when there is tune spread with transverse amplitude in both planes, and Landau damping of longitudinal or transverse oscillations when there is tune spread with longitudinal amplitude only, and when the relevant frequencies in the impedance are small compared to the frequencies in the bunch. The latter has been described by Wang and others $[1,2]$, however, only for Gaussian distributions. Here, we treat distributions other than Gaussian, and point out a caution for the use of these stability diagrams in the transverse case which was not addressed in [2]. These computations require one to ignore azimuthal mode coupling; however, they do demonstrate how to determine the effects of tune spread on mode coupling under some conditions.

First, assume that the coordinates in terms of actionangle variables are $\sqrt{2 J_{\alpha} \beta_{\alpha}(s)} \cos \left[\theta_{\alpha}+\Delta \psi_{\alpha}(s)\right]$, and that the distribution about which one is analyzing perturbations is of the form

$$
\Psi_{0}(\boldsymbol{J})=(2 \pi)^{3} \frac{1}{\epsilon_{x} \epsilon_{y} \epsilon_{z}} S\left(J_{x} / \epsilon_{x}, J_{y} / \epsilon_{y}\right) \lambda\left(J_{z} / \epsilon_{z}\right)
$$

where the integrals of the functions $\lambda$ and $S$ are 1, as are their first moments. Then the following integrals, closely related to the beam transfer function, appear when performing a perturbation analysis to obtain the coherent modes (see, for example, [3]):

$$
\begin{gathered}
-\int \frac{J_{y} \partial \Psi_{0} / \partial J_{y}}{\Omega-\omega_{y}\left(J_{x}, J_{y}\right)-m \omega_{z}\left(J_{x}, J_{y}\right)} d^{3} \boldsymbol{J} \\
\int \frac{J_{z}^{|m|} \Psi_{0}(\boldsymbol{J})}{\Omega-\omega_{y}\left(J_{z}\right)-m \omega_{z}\left(J_{z}\right)} d^{3} \boldsymbol{J} \\
-\int \frac{J_{z}^{|m|} \partial \Psi_{0} / \partial J_{z}}{\Omega-m \omega_{z}\left(J_{z}\right)} d^{3} \boldsymbol{J}
\end{gathered}
$$

where $m$ is the longitudinal azimuthal mode number. These are for the cases of transverse $y$ modes for tune spread with two-plane transverse amplitude (Eq. (1)), transverse $y$ modes for tune spread with longitudinal amplitude (Eq. (2)), and longitudinal modes for tune spread with longitudinal amplitude (Eq. (3)). There are similar terms with $\omega_{y}$ taking the opposite sign. Equations (2-3) are only correct under the assumption that the frequency of the impedance is small compared to the frequencies in the bunch spectrum. In the more general case these terms will depend on the frequency of the impedance. Also, Eqs. (12) ignore a term giving the longitudinal force due to the transverse wake, which is typically small.

If $\boldsymbol{\omega}(\boldsymbol{J})$ is a constant $\boldsymbol{\omega}_{L}$ ("linear lattice"), then the integrals (1-3) simplify greatly:

$$
\begin{gathered}
\frac{1}{\Omega-\omega_{L y}-m \omega_{L z}} \\
\frac{1}{\Omega-\omega_{L y}-m \omega_{L z}} \int J_{z}^{|m|} \Psi_{0}(\boldsymbol{J}) d^{3} \boldsymbol{J} \\
-\frac{1}{\Omega-m \omega_{L z}} \int J_{z}^{|m|} \frac{\partial \Psi_{0}}{\partial J_{z}} d^{3} \boldsymbol{J}
\end{gathered}
$$

When this is the case, the coherent frequencies $\Omega$ can be found by solving an eigenvalue problem. If one ignores azimuthal mode coupling, the problem for the nonlinear lattice can be solved in terms of the problem for the linear lattice: solve the eigenvalue system to find the value of the appropriate term from Eqs. (4-6), equate that to the corresponding term from Eqs. (1-3), and solve for the $\Omega$ appearing in the latter.

Equations (1-3) do not map the complex $\Omega$ plane completely onto itself; there are some points in the complex plane for which there is no $\Omega$ mapped onto that point. This is because the integrals (1-3) are bounded functions of $\Omega$; in other words, for currents/impedances which are sufficiently small, even though there is a solution for the eigenvalue problem for a linear lattice, there is no corresponding solution for the problem for the nonlinear lattice. Such a solution is "Landau damped." 


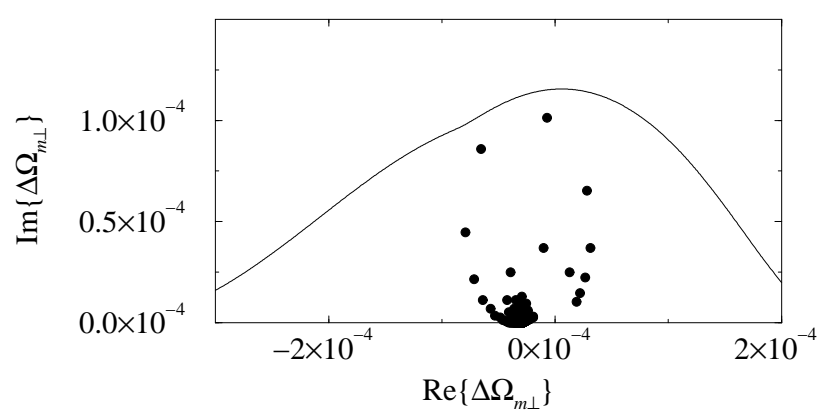

Figure 1: Example use of a stability diagram. The circles are the complex tune shifts of the transverse $m=1$ multibunch modes computed for a linear lattice. The solid line is the stability diagram. In this case, all the modes are Landau damped since they all lie below the stability curve.

A stability diagram is simply a "stability curve" drawn in the plane of the complex coherent tune shifts for the linear problem which delimits the region for which solutions exist for the nonlinear problem. Figure 1 demonstrates how a stability diagram is used.

Formulas and example stability diagrams will be given here; derivations will be given in a forthcoming paper. Programs to produce the curves described here can be obtained from http://wwwslap.cern.ch/collective/jsberg/landau/.

We make the additional assumption that the incoherent tune, due to forces from magnets and potential well distortion, is linear in the action: $\boldsymbol{\omega}=\boldsymbol{\omega}_{L}+A \boldsymbol{J}$. $A$ is a symmetric constant matrix, whose components are given by $A_{\alpha \beta}=a_{\alpha \beta} / \epsilon_{\beta}$, where the $\epsilon_{\beta}$ are the emittances. This is a good assumption in the transverse planes, but may not be as good in the longitudinal plane, mainly because of potential-well distortion.

\section{TRANSVERSE TUNE SPREAD}

For transverse tune spread only, begin by making a further assumption that $S(x, y)=\rho(x+y)$. The integral (1) is

$$
-\int_{0}^{\infty} \int_{0}^{\infty} \frac{y \rho^{\prime}(x+y)}{\Delta \Omega_{m \perp}-v_{x} x-v_{y} y} d x d y
$$

where $\Delta \Omega_{m \perp}=\Omega-\omega_{L y}-m \omega_{L z}$ and $v_{\alpha}=a_{y \alpha}+m a_{z \alpha}$. This integral can be written in the form

$$
\begin{gathered}
\frac{w_{y} f^{\prime}\left(w_{y}\right)-v_{x} \frac{f\left(w_{y}\right)-f\left(w_{x}\right)}{v_{y}-v_{x}}}{v_{y}-v_{x}}, \quad \text { for } v_{x} \neq v_{y} \\
-\frac{1}{2 v_{y}} \frac{d}{d w_{y}}\left[w_{y}^{2} f^{\prime}\left(w_{y}\right)\right] \quad \text { for } v_{x}=v_{y}
\end{gathered}
$$

where $w_{\alpha}=v_{\alpha} / \Delta \Omega_{m \perp}$, and

$$
f(z)=-\int_{0}^{\infty} \rho(u) \ln (1-z u) d u .
$$

The asymptotic expansion of $f(z)$ as $z \rightarrow 0$ is $z+z^{2}+$ $O\left(z^{3}\right)$, making the expansion of the reciprocal of Eq. (7)

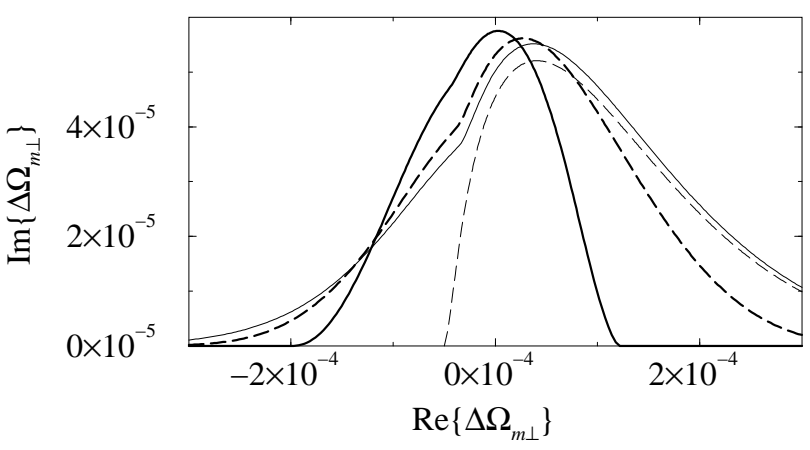

Figure 2: Stability curves for transverse tune spread, when $v_{y}=5 \times 10^{-5}$. The thin lines are for the distribution (8), whereas the thick lines are for (9) ( $\mu=4.5$ for the solid, $\mu=12.5$ for the dashed). All lines have $v_{x}=-3.58 \times$ $10^{-5}$, except for the thin dashed line, for which it is zero (one-dimensional).

for $\Delta \Omega_{m \perp}$ large $\Delta \Omega_{m \perp}-v_{x}-2 v_{y}+O\left(v_{i} / \Delta \Omega_{m \perp}^{2}\right)$. This asymptotic expansion allows one to compute the effects of tune spread when the mode one is examining has a coherent tune shift which is large compared to the tune spread. In such cases, one can compute the mode frequencies to second order in the ratio of the tune spread to the tune shift by replacing all the terms like Eq. (1) with the corresponding term (4), except that $\omega_{L \alpha}+a_{\alpha x}+2 a_{\alpha y}$ is used instead of $\omega_{L \alpha}$, expressing the fact that the average tune in the bunch is not the tune at zero amplitude. This allows one to perform a mode coupling analysis in cases where both modes have shifted by amounts large compared to the tune spread.

\subsection{Specific Distributions}

Consider two distributions

$$
\begin{gathered}
\rho(u)=e^{-u} \\
\rho(u)=\frac{(\mu-1)(\mu-2)}{\mu^{2}}\left(1-\frac{u}{\mu}\right)^{\mu-3}, \quad 0 \leq u \leq \mu
\end{gathered}
$$

which correspond to Gaussian and parabolic-like distributions in one transverse coordinate:

$$
\begin{gathered}
\frac{1}{\sqrt{2 \pi} \sigma_{y}} e^{-y^{2} / 2 \sigma_{y}^{2}} \\
\frac{1}{\sqrt{2 \pi \mu} \sigma_{y}} \frac{\Gamma(\mu)}{\Gamma(\mu-1 / 2)}\left(1-\frac{y^{2}}{2 \mu \sigma_{y}^{2}}\right)^{\mu-3 / 2} \cdot|y|<\sqrt{2 \mu} \sigma_{y}
\end{gathered}
$$

The distribution (11) gives a good approximation for a beam which has been collimated at an amplitude $\sqrt{2 \mu} \sigma_{y}$. The $f(z)$ for (8) and (9) respectively are $-e^{-z^{-1}} E_{1}\left(-z^{-1}\right)$ and $z F(1,1 ; \mu ; z \mu)$, where $E_{1}$ is the exponential integral, and $F$ is a hypergeometric function (which for $2 \mu$ an integer, is expressible in terms of elementary functions in this case and subsequent cases) [4].

Figure 2 demonstrates that including the tune spread in both planes can give substantial improvement over the case 
where only one plane is considered. It also demonstrates that removing the high-amplitude tails from the distribution can substantially affect the amount of Landau damping one obtains.

\section{LONGITUDINAL TUNE SPREAD}

Next, consider Landau damping of either transverse or longitudinal oscillations due to longitudinal tune spread. In this case, one computes

$$
\left[\int_{0}^{\infty} u^{|m|} f(u) d u\right]^{-1} \int_{0}^{\infty} \frac{u^{|m|} f(u) d u}{\Delta \Omega_{m}-v_{z} u}
$$

where the symbols are defined as:

\begin{tabular}{c|cc} 
Symbol & Transverse & Longitudinal \\
\hline$f(u)$ & $\lambda(u)$ & $d \lambda / d u$ \\
$v_{z}$ & $a_{y z}+m a_{z z}$ & $m a_{z z}$ \\
$\Delta \Omega_{m}$ & $\Omega-\omega_{L y}-m \omega_{L z}$ & $\Omega-m \omega_{L z}$
\end{tabular}

and compares the results to $1 / \Delta \Omega_{m}$ for the linear lattice.

\subsection{Specific Distributions}

Expressions can be obtained in the cases when $\lambda(u)$ takes on one of the two forms

$$
\begin{gathered}
e^{-u} \\
\frac{\mu-1}{\mu}\left(1-\frac{u}{\mu}\right)^{\mu-2} .
\end{gathered}
$$

These correspond to distributions in the longitudinal displacement given by Eqs. (10) and (11). For longitudinal oscillations, (12) is

$$
\begin{gathered}
\frac{-1}{\Delta \Omega_{m}}\left[\frac{z^{-|m|-1}}{|m| !} e^{-1 / z} E_{1}(-1 / z)+\sum_{k=1}^{|m|} \frac{(|m|-k) !}{|m| !} z^{-k}\right. \\
\frac{1}{\Delta \Omega_{m}} F(1,|m|+1 ; \mu+|m|-1 ; \mu z)
\end{gathered}
$$

for the two $\lambda$ respectively, where $z=v_{z} / \Delta \Omega_{m}$. For the transverse case, the expression for the exponential distribution is the same, and for the other one obtains $F(1,|m|+$ $1 ; \mu+|m| ; \mu z)$.

The asymptotic expansion of the reciprocal of (12) for large $\Delta \Omega_{m}$ is now given by

$$
\Delta \Omega_{m}-v_{z} \frac{\int_{0}^{\infty} u^{|m|+1} f(u) d u}{\int_{0}^{\infty} u^{|m|} f(u) d u}+O\left(\frac{v_{z}^{2}}{\Delta \Omega_{m}}\right) .
$$

The coefficient of $v_{z}$ is $|m|+1$ for (13), and $\mu(|m|+$ $1) /(\mu+|m|)$ and $\mu(|m|+1) /(\mu+|m|-1)$ for (14) for transverse and longitudinal oscillations respectively. An argument for cases with large tune shifts similar to that which was made for the transverse case applies here as well.

The stability curves for this case look qualitatively similar to those for the transverse case with tune spread in only one plane. However, as $|m|$ increases, the size of the stability region increases rapidly (roughly linearly in $|m|$ ). Also, collimating the distribution gives a more drastic reduction of the stability region than in the transverse case.

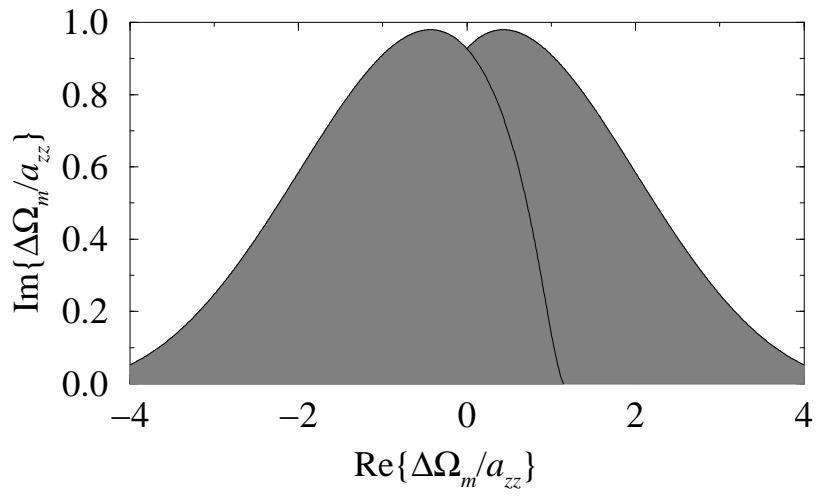

Figure 3: Stability curves for transverse oscillations when $a_{y z}=0$. Vertical lines give the stable region for $m=+1$, and horizontal lines give the stable region for $m=-1$.

\subsection{Other Points of Interest}

To make the problem more tractable, it was assumed that the relevant frequencies in the impedance were small compared to the frequencies in the bunch spectrum. This results in the $J_{z}^{|m|}$ terms in Eqs. (1-6). In the more general case, this factor is replaced by a function which depends on the frequency of the impedance. This can significantly reduce the size of the stability region.

Often one considers transverse oscillations with $a_{y z}=$ 0 . The coherent frequencies one finds are virtually identical for the modes with the same $|m|$ but different signs of $m$. However, the stability curves are mirror reflections of each other about the imaginary axis. Thus, the modes generally need to be within the intersection of the two stability regions, as shown in Fig. 3, often preventing Landau damping due to longitudinal tune spread when there are large real tune shifts in the transverse modes.

Combining these facts with the observation that the longitudinal tune shift with amplitude is often very nonlinear in action due to potential-well distortion, one should use a great deal of caution in relying on stability diagram based models for determining Landau damping due to longitudinal tune spread.

\section{REFERENCES}

[1] J. M. Wang, "Longitudinal Symmetric Coupled Bunch Modes," Brookhaven National Laboratory report BNL 51302, Upton, New York, 1980.

[2] M. S. Zisman, S. Chattopadhyay, and J. J. Bisognano, “ZAP User's Manual," Lawrence Berkeley Laboratory report LBL-21270, Berkeley, California, 1986.

[3] J. S. Berg, "Coherent Modes for Multiple Non-Rigid Bunches in a Storage Ring," Ph.D. thesis, Stanford University, 1996.

[4] M. Abramowitz and I. A. Stegun, Handbook of Mathematical Functions (Dover, New York, 1972). 